\title{
Urinary excretion of intermediate pathogenic leptospires among "renal patients" of Girandurukotte, Sri Lanka: Interim results
}

\author{
GRA Fonseka ${ }^{1}$, P Senarathne ${ }^{1}$, HMRM Thilakarathne ${ }^{1}$, AKUI Karunadasa ${ }^{1}$, \\ YD Sarathkumara $^{1}$, BDS Muthusinghe ${ }^{2}$, S Lokupathirage ${ }^{3}$, A Kumara ${ }^{1}$, \\ $\mathrm{N}_{\text {Nanayakkara }}{ }^{4}$ L Gunaratne ${ }^{5}$, K Yoshimatsu ${ }^{2,3}, \mathrm{~N} \mathrm{Koizumi}^{6}$, CD Gamage ${ }^{1}$
}

Introduction and Objectives: Chronic kidney disease (CKD) and CKD of uncertain aetiology (CKDu) pose a major public health threat in Sri Lanka. Untreated asymptomatic leptospirosiscould progress to a chronic infection, whereby leptospires colonize the renal tubulesand are shed in urine concomitantly. This leads to decreased renal functionality which could progress to CKD/CKDu. The farmers of Sri Lanka who are a high-risk group for leptospirosis,are affected by CKD/CKDu. Thus we hypothesize that leptospirosis could be a confounding factor between $\mathrm{CKD} / \mathrm{CKDu}$ and farming. The aim of this study was to detect urinary excretion of leptospires among renal patients living in Girandurukotte, Sri Lanka.

Methods: Urine (50 mL) and demographic data were collected from clinically diagnosed $\mathrm{CKD} / \mathrm{CKDu}$ patients $(\mathrm{n}=30)$ from the Girandurukotte District Hospital, Badulla. Urine samples were filtered on site, using a $0.22 \mu \mathrm{m}$ filter unit. The filter paper was deposited in tubes containing $1.5 \mathrm{ml}$ water and placed on a shaker overnight, to enable detachment of Leptospira cells from the filter paper. The tubes were vortexed at high speed for 30 seconds the next day, following which DNA extraction was performed as per the manufacturer's protocol (Qiagen ${ }^{\circledR D N A e a s y b l o o d ~ a n d ~ t i s s u e ~ k i t) . ~ P C R ~ w a s ~ c a r r i e d ~ o u t ~ t o ~ d e t e c t ~ p a t h o g e n i c ~}$ and intermediate pathogenic flaBgenes. PCR positive samples were sent to National Institute of Infectious Diseases, Japan for sequencing and phylogenetic analysis was done using flaB sequences obtained.

Results: Of the 30 renal patient samples that were subjected to PCR, none of the samples were positive for pathogenic flaB PCR, while one sample was positive for intermediate pathogenic flaB PCR. Positive amplicon sequence showed98\% identity to L. licerasiae.

Conclusions: Asymptomatic renal colonization by pathogenic and intermediate pathogenic Leptospiraspp. has been observed in endemic regions. However, this is the first report in Sri Lanka where an intermediate pathogenic Leptospira spp. was found to colonize the kidneys asymptomatically, despite leptospirosis being a notifiable disease since 1991. These results do not show any association between leptospirosis and CKD/CKDu.

Keywords: Leptospirosis, CKDu, PCR, phylogenetic analysis

Funding: University of Peradeniya (2018/28M) \& NSF, Sri Lanka (RPHS/2016/CKDu/06).

\footnotetext{
${ }^{1} 1$ Department of Microbiology, Faculty of Medicine, University of Peradeniya, Sri Lanka

${ }^{2}$ Graduate School of Infectious Diseases, Hokkaido University, Japan

${ }^{3}$ Department of Microbiology and Immunology, Faculty of Medicine, Hokaido University, Japan

${ }^{4}$ Nephrology and Transplantation Unit, National Hospital, Kandy, Sri Lanka

${ }^{5}$ Renal Unit, District Hospital, Girandurukotte, Sri Lanka

${ }^{6}$ Department of Bacteriology, National Institute of Infectious Diseases, Tokyo, Japan
}

Address for correspondence: Prof. CD Gamage. Telephone: +94771661460

Email: chandika.gamage@med.pdn.ac.lk (D) https://orcid.org/0000-0003-0974-5730 